\title{
Bone Mineral Density in Children and Adolescents with Perinatal HIV Infection
}

\author{
Linda A. Dimeglio, MD, MPH ${ }^{1}$, JiaJia Wang, MS $^{2}$, George K. Siberry, MD, MPH ${ }^{3}$, Tracie L. \\ Miller, MD ${ }^{4}$, Mitchell E. Geffner, MD $^{5}$, Rohan Hazra, MD $^{3}$, William Borkowsky, MD ${ }^{6}$, Janet S. \\ Chen, MD ${ }^{7}$, Laurie Dooley, MT, MBA ${ }^{8}$, Kunjal Patel, DSc, MPH ${ }^{9}$, Russell B. Van Dyke, MD $^{10}$, \\ Roger A. Fielding, PhD ${ }^{11}$, Yared Gurmu, MA ${ }^{12}$, Denise L. Jacobson, PhD, MPH $^{2}$, and for the \\ Pediatric HIV/AIDS Cohort Study \\ ${ }^{1}$ Section of Pediatric Endocrinology and Diabetology, Department of Pediatrics, Indiana, \\ University School of Medicine, Indianapolis, IN \\ ${ }^{2}$ Center for Biostatistics in AIDS Research, Harvard School of Public Health, Boston, MA \\ ${ }^{3}$ Pediatric Adolescent Maternal AIDS Branch, Eunice Kennedy Shriver National Institute of Child \\ Health and Human Development, National Institutes of Health, Bethesda, MD \\ ${ }^{4}$ Division of Pediatric Clinical Research, Department of Pediatrics, Miller School of Medicine at \\ the University of Miami, Miami, FL \\ ${ }^{5}$ Saban Research Institute, Children's Hospital Los Angeles, Los Angeles, CA \\ ${ }^{6}$ New York University Langone Medical Center, New York, NY \\ ${ }^{7}$ Department of Pediatrics, Drexel University College of Medicine, Philadelphia, PA \\ ${ }^{8}$ Frontier Science and Technology Research Foundation, Amherst, NY \\ ${ }^{9}$ Department of Epidemiology, Harvard School of Public Health, Boston, MA \\ ${ }^{10}$ Department of Pediatrics, Tulane University Health Sciences Center, New Orleans, LA \\ ${ }^{11}$ Body Composition Analysis Center, Friedman School of Nutrition Science and Policy, Tufts \\ University, Boston, MA \\ ${ }^{12}$ Department of Biostatistics, Harvard School of Public Health, Boston, MA
}

\begin{abstract}
Corresponding Author/Requests for Reprints: Linda DiMeglio, MD, MPH, Room 5960, 705 Riley Hospital Drive, Indianapolis, IN 46202-5225, dimeglio@iupui.edu.

All authors declare no conflict of interest.

Disclaimer: The conclusions and opinions expressed in this article are those of the authors and do not necessarily reflect those of the National Institutes of Health or US Department of Health and Human Services.

Author Contributions: RBV, DLJ, MEG, and TLM were the primary authors who conceived of and designed the study. LAD and DLJ led the writing of the manuscript. LAD, JW, DLJ, GKS, TLM, RH, and RBV were primarily responsible for analysis and interpretation of the data. KP, RAF, LD and YG assisted with data interpretation in their individual areas of expertise. WB and JSC were involved in the conduct of the trial and provided key feedback on the MS content. All authors were involved in the design and conduct of the PHACS protocol, interpretation of results, and revised the manuscript critically.
\end{abstract}


Objective-To estimate prevalence of low bone mineral density (BMD) in perinatally HIV infected (HIV+) and HIV-exposed but uninfected (HEU) children, and to determine predictors of $\mathrm{BMD}$ in $\mathrm{HIV}+$.

Design-Cross-sectional analysis within a 15-site United States and Puerto Rico cohort study.

Methods-Total body (TB) and lumbar spine (LS) BMD were measured using dual energy-xray absorptiometry. BMD Z-scores accounted for bone age and sex. Multiple linear regression was used to evaluate differences in Z-scores by HIV status and for predictors of BMD in HIV+.

Results-350 HIV+ and $160 \mathrm{HEU}$ were enrolled. Mean age was 12.6 and 10.7 years for HIV+ and HEU, respectively. Most (87\%) HIV+ were receiving highly active antiretroviral therapy (HAART). More HIV+ than HEU had TB and LS Z-scores < -2.0 (TB: $7 \%$ vs. 1\%, p=0.008; LS: $4 \%$ vs. $1 \%, \mathrm{p}=0.08$ ). Average differences in $\mathrm{Z}$-scores between HIV+ and HEU were attenuated after height and/or weight adjustment. Among HIV+, TB Z-scores were lower in those with higher $\mathrm{CD} 4 \%$ and in those who ever used boosted protease inhibitors or lamivudine. LS Z-scores were lower with higher peak viral load and CD4\%, more years on HAART, and ever use of indinavir.

Conclusions-Rates of low BMD in HIV+ children were greater than expected based on normal population distributions. These differences were partially explained by delays in growth. Since most HIV+ children in this study had not entered their pubertal growth spurt, prepubertal factors associated with BMD, magnified or carried forward, may result in sub-optimal peak BMD in adulthood.

\section{Keywords}

Anti-retroviral agents; Bone age; Bone mineral density; HIV; Children; CD4; Viral load

\section{Introduction}

Highly active antiretroviral therapy (HAART) has led to dramatic declines in morbidity and mortality for HIV-infected persons. However, this improved lifespan has been accompanied by greater recognition of long-term complications of the disease and its therapies, including adverse bone health effects [1]. Low bone mineral density (BMD) is common in HIVinfected adults $[2,3]$. A recent meta-analysis demonstrated a $15 \%$ prevalence of osteoporosis and a 52\% prevalence of osteopenia in HIV-infected adults [4]. For children with perinatal HIV infection (HIV+), adverse bone health effects can be magnified by the life-long exposure to both HIV infection and its treatment. These effects may be particularly important during puberty when children experience the most rapid growth and bone mineral accrual [5]. Perinatally infected adolescents (particularly boys) have lower BMD at the end of puberty than do their HIV-uninfected and unexposed peers [6], which may lead to lower adult peak bone mass and subsequently contribute to increased fracture rates [7, 8].

Low BMD in HIV-infected individuals may be the result of chronic inflammation and promotion of osteoblast apoptosis and osteoclast proliferation by the HIV envelope glycoprotein, gp120 [9, 10]. Other purported factors include HIV-associated complications such as wasting, corticosteroid use, hypogonadism, renal disease, and adverse effects of anti-retroviral therapy (ART) [11]. Finally, traditional risk factors for osteoporosis, such as 
older age, female gender, low body mass index (BMI), diabetes, decreased physical activity, vitamin D deficiency, smoking, and alcohol use may also play similar roles in HIV-infected individuals as they do in uninfected individuals.

We measured BMD in children with perinatal HIV infection and children who were perinatally HIV-exposed but uninfected (HEU) to examine the prevalence of low total body (TB) and/or lumbar spine (LS) BMD in the current HAART era. We hypothesized that HIV + children would have lower BMD than expected when compared to both age- and sexmatched normative data and to their HEU peers. We sought to determine which factors, including lifestyle, disease and treatment characteristics, were associated with BMD outcomes in the HIV+ children.

\section{Methods}

Children 7-15 years of age born of HIV+ mothers were enrolled into the Adolescent Master Protocol (AMP) of the NIH-supported Pediatric HIV/AIDS Cohort Study (PHACS). AMP is an ongoing prospective cohort study examining specific outcomes of HIV infection and ART in HIV+ pre-adolescents and adolescents. HEU children were enrolled as a comparison group. Enrollment occurred between March 2007 and December 2009 at 15 sites in the United States. Study visits occurred semi-annually through July 2010 and annually thereafter. Institutional Review Boards at all clinical sites and the Harvard School of Public Health approved the protocol. Informed consent from the parent(s) or guardian(s) and assent from the participants were obtained.

\section{Data Collection}

Sociodemographic and clinical history-Sociodemographic characteristics were collected at entry. Clinical data were abstracted from medical records and obtained from prior study databases. Weights and heights were measured and BMI's were calculated [weight $(\mathrm{kg}) /$ height $\left(\mathrm{m}^{2}\right)$ ] and expressed as Z-scores based on CDC growth data [12] as previously described [13]. Trained examiners performed Tanner pubertal staging for breasts (in girls), genitalia, and pubic hair. For HIV+ children, ART start and stop dates, quantitative plasma HIV-1 RNA (copies/mL) (viral load), absolute CD4+ lymphocyte (CD4) count (cells $/ \mathrm{mm}^{3}$ ) and CD4\%, and Centers for Disease Control (CDC) pediatric HIV clinical classification [14] were obtained at each visit from the clinical charts.

Dual-energy X-ray absorptiometry scans (DXA)—TB (including head) BMD and LS BMD were measured for HIV+ and HEU using Lunar (General Electric Healthcare, UK) or Hologic (Hologic Inc., Bedford, MA) DXA scanners according to standard methods. Sites were standardized using circulated phantoms. Scans were sent to the Body Composition Analysis Center at Tufts University School of Medicine for central analysis and standardization. Hologic scans were analyzed using Hologic QDR version 12.3 and APEX version 3.3. Lunar scans were analyzed using Prodigy Advance enCORE 2005 version 9 and enCORE 2011 version 13.6. All scans were analyzed by a single technician blinded to the participants' HIV status. For this analysis, we used the first DXA obtained on study for every participant who had a DXA performed. At the time of the DXA, children who had not yet reached Tanner 5 pubertal status had a left hand and wrist radiograph performed to 
determine bone age (BA) [15]. Normative BMD data from Baylor University [16] were used to generate chronologic age- and sex-adjusted Z-scores (CA-adjusted BMD Z-scores) for TB and LS BMD. In addition, a second set of Z-scores (BA-adjusted BMD Z-scores) was calculated for these two outcomes based on the following algorithm. For children at Tanner stage 1-4, the BA was used instead of the chronological age (CA) if the child's CA was > 1 SD from BA; otherwise the CA was used. For children at Tanner 5, the CA was used. We excluded children at Tanner 1-4 from all analyses if their bone age were missing. The prevalences of BMD Z-scores below $<-1,<-1.5$, and $<-2$ (not mutually exclusive) were calculated for each CA- and BA-adjusted BMD outcome.

Dietary recall and physical activity-Dietary intake and physical activity were assessed by recall using the Block Dietary Questionnaire and the Physical Activity Screener for children and adolescents from Block Dietary Data Systems (Nutriquest, Berkeley, CA). The recommended dietary allowance (RDA) for calcium and vitamin D were based on the Dietary Reference Intakes (Dietary Reference Intakes: The Essential Reference for Dietary Planning and Assessment. 2006 Food and Nutrition Board, Institute of Medicine). Calculated minutes of vigorous physical activity were dichotomized into above or below the $75^{\text {th }}$ percentile, based on the distribution of all HIV+ and HEU.

Statistical Methods-The analysis dataset consisted of children with complete data on TB and/or LS BMD, a bone age if required, and a Tanner stage measurement near the time of DXA. Children's characteristics and prevalence of low BMD by HIV status were compared via Fisher's exact test for categorical variables and Wilcoxon test for continuous variables. Multivariate linear regression models were fit to determine differences by HIV status in BA-adjusted TB and LS BMD Z-scores. We used BA-adjusted values to reduce the effect of variation in bone maturity on outcomes. Sex, race/ethnicity, and Tanner stage were included in all models. Other potential confounders were included in the model if they were significant at $\mathrm{p}<0.1$. The potential confounders included Z-scores for height, weight, any supplement use, vitamin D intake $<$ RDA, and vigorous activity $>75^{\text {th }}$ percentile. When the F-test overall $\mathrm{p}$ value was $<0.05$ for any multi-level categorical variable, pairwise differences were tested by the Wald test and shown when $\mathrm{p} \unlhd$ \.05. Interaction between HIV status and Tanner stage and HIV and sex were tested for each outcome. Among HIV+ children, basic models were fit using linear regression for each variable on each BAadjusted BMD outcome, adjusted for the basic covariates including sex, race/ethnicity, and Tanner stage. The following variables were examined: ART class and individual ART medications, as well as nadir and current CD4\% $(<15 \%, 15-24 \%, \geq 25 \%)$, peak and current viral load, CDC disease classification at the time of DXA, Z-scores for height and weight, any supplement use, vitamin D intake $<\mathrm{RDA}$, and vigorous activity $>75^{\text {th }}$ percentile. Variables associated with the outcomes at $\mathrm{p}<0.2$ in these basic models were tested in multivariate linear regression models to determine independent predictors of BA-adjusted BMD Z-scores in predictive models. Sex, race/ethnicity, and Tanner stage were included in all final models plus any of the above other covariates significant at $\mathrm{p}<0.1$. 


\section{Results}

\section{Characteristics of HIV+ and HEU children}

Of $451 \mathrm{HIV}+$ children and $227 \mathrm{HEU}$ children enrolled in AMP, 613 (350 HIV+ and 160 HEU) had TB and/or LS BMD and a BA available by January 1, 2011 (Table 1). There were no significant differences between those included and those excluded because of a missing BA by CA, sex, race/ethnicity, or Tanner stage. HIV+ children were older than HEU children. The two groups had a similar sex distribution, but the HIV+ children were more likely to be non-Hispanic black. As expected, because of their older age, HIV+ had more advanced sexual maturation than HEU and slightly older BA. HIV+ had lower height, weight, and BMI z-scores. HEU children were more likely than HIV+ to have advanced BA compared to $\mathrm{HIV}+(34 \%$ vs. $16 \%)$.

Data on vitamin D and calcium consumption were available from 457 participants (320 HIV + and $137 \mathrm{HEU}$ ) (Table 1). Intake from dietary sources was similar between the groups, but HIV+ children were more likely to report use of dietary supplements. HIV+ were less likely than HEU to be below the dietary reference intake for vitamin D (52\% vs. 59\%), but not calcium ( $73 \%$ vs. $67 \%$ ). HIV+ and HEU reported similar levels of vigorous physical activity.

\section{HIV disease severity and ART}

Table 2 shows HIV-specific disease characteristics of HIV+ children at the time of DXA. Twenty-five percent were CDC clinical category $\mathrm{C}$ and their median CD4 count and nadir $\mathrm{CD} 4 \%$ at the time of DXA were 725 cells $/ \mathrm{mm}^{3}$ and 33\%, respectively. Fifty-five percent had viral load $\leq 400$ copies $/ \mathrm{mL}$. Eighty-seven percent were receiving HAART, with protease inhibitor (PI)-based regimens being most common (70\%); 55\% of the HIV+ children were receiving a ritonavir-boosted PI and an additional $9 \%$ had previously received a boosted PI. The median lifetime duration of HAART was 9.5 (interquartile range: 7.1, 11.3) years. The most common nucleoside reverse transcriptase inhibitors (NRTIs) ever used (previously and/or at the time of DXA) were lamivudine (3TC) (90\%), zidovudine (ZDV) (85\%), and stavudine (76\%). Tenofovir disoproxil fumarate (TDF) was used at the time of the DXA by $21 \%$, with an additional $4 \%$ having received it previously. Thirty-five percent of children had ever used efavirenz and $35 \%$ nevirapine.

\section{Unadjusted prevalence of low BMD in HIV+ and HEU based on different cut-offs for CA- and BA-adjusted BMD Z-scores}

Table 3 shows the percentage of HIV+ and HEU children with TB and LS BMD Z-scores < $-1.0,<-1.5$, and $<-2.0 \mathrm{SD}$ (categories not mutually exclusive). The results on the left show CA-adjusted BMD Z-scores and those on the right show BA-adjusted BMD Z-scores, as specified in the Methods. For TB BMD, the difference in proportion between HIV+ and HEU for each cut-off ranged from 5-6\% higher in HIV+ for the CA-adjusted and 3-6\% higher for the BA-adjusted Z-scores. Compared to HEU, HIV+ had a greater proportion of children with CA- or BA-adjusted TB Z-score $<-2.0(\mathrm{p}=0.019$ and $\mathrm{p}=0.008$, respectively). For LS BMD, the difference in proportion between HIV+ and HEU at each cut-off ranged from 3-7\% higher in HIV+ for CA- and 3-5\% higher for BA-adjusted Z- 
scores compared to HEU. The differences were not statistically significant at $p<0.05$ for any cut-off of LS, but the trends suggest a greater proportion of HIV+ than HEU were below all cut-offs for the CA-adjusted LS Z-scores and at the $<-2.0$ cut-off for BA-adjusted LS Zscore.

\section{Comparison of TB and spine BMD Z-scores between HIV+ and HEU children}

The mean (SD) TB Z-scores in HIV+ and HEU were -0.06 (1.3) vs. 0.40 (1.4) for CAadjusted TB and -0.13 (1.2) vs. 0.03 (1.2) for BA-adjusted TB. Mean (SD) LS Z-scores for HIV+ and HEU were 0.06 (1.3) vs. 0.53 (1.5) for CA-adjusted LS and -0.03 (1.2) vs. 0.16 (1.3) for BA-adjusted LS.

Table 4 shows the univariate (unadjusted) and multivariate models of linear regression analyses on BA-adjusted BMD outcomes. The unadjusted difference between HIV+ and HEU was -0.16 for TB and -0.19 for LS. With adjustment for sex, race/ethnicity, and Tanner stage (not shown), the difference between the two groups was -0.33 ( $\mathrm{p}=0.004)$ for TB and $-0.30(\mathrm{p}=0.01)$ for LS. However, with further adjustment for height and weight as shown in the multivariate models in Table 4, these differences between HIV+ and HEU were attenuated for both TB $(-0.03, \mathrm{p}=0.81)$ and LS $(0.03, \mathrm{p}=0.79)$. In the multivariate models, TB and LS Z-scores were associated with race/ethnicity, Tanner stage, height, and weight, but not sex. There was no interaction of sex or Tanner by HIV status on TB or LS BMD. Weight was a better predictor of outcomes than was BMI.

\section{Correlates of spine and TB BMD in HIV+ children}

In HIV+ children, each ARV was regressed individually on BA-adjusted TB and LS BMD Z-scores, adjusted for sex, race/ethnicity, and Tanner stage. The results are described for "ever use" of the ARVs with a p value $<0.2$ as well as for years on HAART. In univariate analyses, BA-adjusted TB BMD was associated with ever use of PI $(-0.25, \mathrm{p}=0.16)$; boosted PI (-0.27, $\mathrm{p}=0.03)$, lamivudine $(-0.73, \mathrm{p}<0.001)$; indinavir $(-0.40, \mathrm{p}=0.10)$; and TDF $(-0.24$, $\mathrm{p}=0.17)$. BA-adjusted LS BMD was associated with ever use of NNRTI (0.21, $\mathrm{p}=0.09)$; PI $(-0.23, \mathrm{p}=0.20)$; boosted PI $(-0.32, \mathrm{p}=0.009)$; zidovudine $(-0.55, \mathrm{p}=0.006)$; indinavir $(-0.36$, $\mathrm{p}=0.13$. $)$ TDF $(-0.26, \mathrm{p}=0.13)$; and years on HAART $(-0.03 / \mathrm{yr}, \mathrm{p}=0.14)$.

The multivariate results among HIV+ children are shown in Table 5. BA-adjusted TB BMD Z-scores were higher in Non-Hispanic blacks, Tanner 4 and 5 vs. Tanner 1, and with greater height and weight $Z$-scores. They were lower with CD4\% $\geq 25 \%$ and with ever use of lamivudine. They did not differ by sex. BA-adjusted LS BMD Z-scores were higher with greater weight Z-score and more minutes of vigorous physical activity. They were lower with CD4\% greater than $15 \%$, peak viral load $>10,000$ copies $/ \mathrm{mL}$, greater duration of HAART, and indinavir use. LS did not differ by sex, race/ethnicity, Tanner stage, or height. No other ARVs were significant in the multivariate models for either outcome.

\section{Discussion}

In this large cross-sectional study, we found lower TB and LS BMD Z-scores in HIV+ compared to HEU children. Some, but not all, of the decrease in BMD was explained by differences in bone maturation between HIV+ and HEU children, since adjustment for BA 
attenuated, but did not eliminate, the differences. Whether CA- or BA-adjusted, there were relatively few HIV+ children with low BMD (Z-score < - 2); however, we observed still more than double the expected rates based on normal population distributions. We found that the differences in BMD between the HIV+ and HEU groups, both at the TB and LS, persisted after adjusting for sex, race, and pubertal maturity, but were greatly attenuated and no longer significant after adjustment for height, weight, or BMI Z-score.

Some of the differences between cohorts may have been due to artifactual effects of bone size on areal BMD measures, since our HIV+ children were shorter and, therefore, likely had lower areal BMD by DXA. Since areal BMD $\left(\mathrm{g} / \mathrm{cm}^{2}\right)$ is a two-dimensional measurement derived by dividing bone mineral content $(\mathrm{g})$ by the apparent area $\left(\mathrm{cm}^{2}\right)$ of the analyzed bone, it provides a two-dimensional estimate of a three-dimensional structure, which is dependent on the size of the bone. Some of the difference may also have been due to lighter children having lower muscle mass exerting force on bone, resulting in lower bone mass, since the difference was also mediated by weight [17]. Differences in body weight in HIV+ adults largely explain differences in their BMDs [18]. Unlike a prior study, which reported greater differences in BMD between HIV+ and uninfected children with increasing Tanner stage, we did not find any difference in BMD between HIV+ and HEU across Tanner stages [6]. This may be because our population was younger or because our controls were HEU, while the other study's controls were HIV-unexposed.

It is possible that we underestimated our rates of low BMD for age and sex due to differences between the racial composition of our cohort and that of the Baylor normative data set used to determine Z-scores in our cohort [16]. Blacks have higher BMD than do whites [19]. Sixty-six percent of our HIV+ and 53\% of our HEU population were black, non-Hispanic compared to fewer than one-third of the subjects in the Baylor normative data. However, when we repeated our analyses using Hologic reference data (which account for race, in addition to sex and age [18]) in the subset of participants (166 HIV+ and $90 \mathrm{HEU}$ ) with DXA by Hologic scanners, the findings for the overall prevalence of low BMD Zscores by HIV status were similar, and BMD differences between HIV+ and HEU were still attenuated after adjustment for height Z-score.

Without fracture data in this HIV+ cohort, we are unable to evaluate whether low BMD scores are associated with bone fractures or fragility (osteoporosis) [20]. Several reports suggest higher fracture rates in HIV+ adults [8,21-23] and some have speculated that HIV+ children may be at higher risk of fracture than their uninfected peers, but the presence, magnitude, and timing of this increased risk are not certain [24]. Although we assed vitamin D intake, we also did not have blood measures of 25-hydroxy vitamin D status.

For the HIV+ children, specific ARVs and measures of disease severity were associated with BMD. For example, ever use of boosted PI or lamivudine and higher CD4\% were associated with a lower TB BMD, while longer duration of HAART, ever use of indinavir, higher CD4\%, and higher peak viral load were associated with a lower LS BMD. The associations of greater height and/or weight Z-scores with TB and LS Z-scores within the $\mathrm{HIV}+$ cohort could be expected to be an indicator of differences in past degree of disease 
severity, although there were no differences by CDC stage and nadir CD4, which also reflect past disease severity.

Data from studies examining the effects of ART on bone in HIV+ individuals are conflicting, but, in general, suggest that initiation of HAART is associated with early bone loss. HAART-treated HIV-infected adults have a 2.4-fold increased risk of osteoporosis vs. those who are HIV-infected, but HAART-naïve [2]. In addition, adults randomized to continuous HAART were at risk of greater BMD loss and possibly of fracture than were adults randomized to intermittent, CD4-guided HAART [11]. In our study, 87\% of HIV+ children were receiving HAART, mostly PI-based. Although interpretation of these effects in children is confounded by their relatively wide range of age and pubertal status, the observed negative effects of boosted PI, lamivudine, and indinavir use on BMD are consistent with findings in other published studies. Previous reports suggest that boosted PI is associated with lower bone mineral content and TB BMD [6]. Others have found adverse bone effects associated with regimens containing lamivudine and zidovudine [25, 26]. Indinavir is known to increase osteoclast activity [27]. The positive effect of vigorous physical activity on spine BMD is consistent with findings in other pediatric studies [28] and is of import, as physical activity is a modifiable risk factor.

We had expected to see lower BMD with TDF use, but did not. Pediatric and adult studies of TDF-containing HAART treatment have shown BMD decreases, but the effect of chronic TDF-containing therapy, especially in adolescents, is still unclear [30]. Only 96 (23\%) HIV+ children in the current study had ever used TDF and only $\sim 1 / 3$ had $>1$ year of TDF exposure before the age of 12 . The children who had ever used TDF did not differ by race or sex from non-users, but were, on average, 2.1 years older than those who had never received TDF. This pattern of use is not surprising, because many children in this relatively young cohort had not reached the age at which guidelines routinely recommend TDF [29].

Evidence that HIV infection itself contributes to bone loss includes data from an HIV-1 transgenic rat model that demonstrates increased osteoclastic bone resorption leading to BMD loss [31]. These findings are supported by data demonstrating high levels of bone turnover markers in post-menopausal HIV-infected women [32]. Lower nadir CD4 counts have also been associated with increased fracture risk [33]. Limited pediatric data suggest that low bone formation (as assessed by serum osteocalcin) may be an initial sign of poor bone status in children with HIV infection [34].

There were relatively low overall rates of reduced BMD for age and sex in our HIV+ cohort, and the differences between HIV+ and HEU were largely attributable to differences in their body size. However, the majority of these children had not yet entered their pubertal growth spurt. Bone deficits may be magnified during the pubertal years, resulting in a failure to reach optimal peak bone mass leading to a trajectory favoring a higher adulthood risk of osteoporosis and increased fractures. This is important given that fracture rates in adults, including adults with HIV infection, are greater with increasing age [8]. This concern about bone health is analogous to concerns about other aspects of long-term health for HIV+ children (e.g., cardiovascular events, renal failure, and premature cognitive decline) where clinical problems with roots in childhood may not become manifest until adulthood. 
Longitudinal studies of bone accrual in a pediatric cohort are critical in order to clarify risk factors and periods of greatest risk for poor bone accrual. It is essential to monitor long-term bone health in this population.

\section{Acknowledgments}

We thank the children and families for their participation in PHACS, and the individuals and institutions involved in the conduct of PHACS.

Funding: The study was supported by the Eunice Kennedy Shriver National Institute of Child Health and Human Development with co-funding from the National Institute of Allergy and Infectious Diseases, National Institute on Drug Abuse, the National Institute of Mental Health, the National Institute on Deafness and Other Communication Disorders, the National Heart Lung and Blood Institute, the National Institute on Alcohol Abuse and Alcoholism, the National Institute of Neurological Disorders and Stroke, the National Institute of Dental and Craniofacial Research, and the Office of AIDS Research through cooperative agreements with the Harvard University School of Public Health (HD052102, 3 U01 HD052102-05S1, 3 U01 HD052102-06S3) (Principal Investigator: George Seage; Project Director: Julie Alperen) and the Tulane University School of Medicine (HD052104, 3U01HD052104-06S1) (Principal Investigator: Russell Van Dyke; Co-Principal Investigator: Kenneth Rich; Project Director: Patrick Davis). Data management services were provided by Frontier Science and Technology Research Foundation (PI: Suzanne Siminski), and regulatory services and logistical support were provided by Westat, Inc. (PI: Julie Davidson).

The following institutions, clinical site investigators, and staff participated in conducting PHACS AMP in 2010, in alphabetical order: Baylor College of Medicine: William Shearer, Mary Paul, Norma Cooper, Lynette Harris; Bronx Lebanon Hospital Center: Murli Purswani, Mahboobullah Baig, Anna Cintron; Children's Diagnostic \& Treatment Center: Ana Puga, Sandra Navarro, Doyle Patton, Deyana Leon; Children's Hospital, Boston: Sandra Burchett, Nancy Karthas, Betsy Kammerer; Children's Memorial Hospital: Ram Yogev, Margaret Ann Sanders, Kathleen Malee, Scott Hunter; Jacobi Medical Center: Andrew Wiznia, Marlene Burey, Molly Nozyce; St. Christopher's Hospital for Children: Janet Chen, Latreca Ivey, Maria Garcia Bulkley, Mitzie Grant; St. Jude Children's Research Hospital: Katherine Knapp, Kim Allison, Megan Wilkins; San Juan Hospital/Department of Pediatrics: Midnela Acevedo-Flores, Heida Rios, Vivian Olivera; Tulane University Health Sciences Center: Margarita Silio, Medea Jones, Patricia Sirois; University of California, San Diego: Stephen Spector, Kim Norris, Sharon Nichols; University of Colorado Denver Health Sciences Center: Elizabeth McFarland, Emily Barr, Robin McEvoy; University of Maryland, Baltimore: Douglas Watson, Nicole Messenger, Rose Belanger; University of Medicine and Dentistry of New Jersey: Arry Dieudonne, Linda Bettica, Susan Adubato; University of Miami: Gwendolyn Scott, Patricia Bryan, Elizabeth Willen. Body Composition Analysis Center at Tufts University: Andrea Desilets, Justin Wheeler.

\section{Reference List}

1. Hazra R, Siberry GK, Mofenson LM. Growing up with HIV: children, adolescents, and young adults with perinatally acquired HIV infection. Annu Rev Med. 2010; 61:169-185. [PubMed: 19622036]

2. Brown TT, Qaqish RB. Antiretroviral therapy and the prevalence of osteopenia and osteoporosis: a meta-analytic review. AIDS. 2006; 20(17):2165-2174. [PubMed: 17086056]

3. McComsey GA, Tebas P, Shane E, Yin MT, Overton ET, Huang JS, et al. Bone disease in HIV infection: a practical review and recommendations for HIV care providers. Clin Infect Dis. 2010; 51(8):937-946. [PubMed: 20839968]

4. Paccou J, Viget N, Legrout-Gerot I, Yazdanpanah Y, Cortet B. Bone loss in patients with HIV infection. Joint Bone Spine. 2009; 76(6):637-641. [PubMed: 19945322]

5. McKay H, Bailey D, Mirwald R, Davison K, Faulkner R. Peak bone mineral accrual and age at menarche in adolescent girls: a 6-year longitudinal study. J Pediatr. 1998; 133(5):682-7. [PubMed: 9821429]

6. Jacobson DL, Lindsey JC, Gordon CM, Moye J, Hardin DS, Mulligan K, et al. Total body and spinal bone mineral density across Tanner stage in perinatally HIV-infected and uninfected children and youth in PACTG 1045. AIDS. 2010; 24(5):687-696. [PubMed: 20168204]

7. Heaney RP, Abrams S, Dawson-Hughes B, Looker A, Marcus R, Matkovic V, et al. Peak bone mass. Osteoporosis International. 2000; 11(12):985-1009. [PubMed: 11256898] 
8. Triant VA, Brown TT, Lee H, Grinspoon SK. Fracture prevalence among human immunodeficiency virus (HIV)-infected versus non-HIV-infected patients in a large U.S. healthcare system. J Clin Endocrinol Metab. 2008; 93(9):3499-3504. [PubMed: 18593764]

9. Fakruddin JM, Laurence J. Interactions among human immunodeficiency virus (HIV)-1, interferongamma and receptor of activated NF-kappa B ligand (RANKL): implications for HIV pathogenesis. Clin Exp Immunol. 2004; 137(3):538-545. [PubMed: 15320903]

10. Gibellini D, De CE, Ponti C, Cimatti L, Borderi M, Tschon M, et al. HIV-1 triggers apoptosis in primary osteoblasts and HOBIT cells through TNFalpha activation. J Med Virol. 2008; 80(9): 1507-1514. [PubMed: 18649336]

11. Grund B, Peng G, Gibert CL, Hoy JF, Isaksson RL, Shlay JC, et al. Continuous antiretroviral therapy decreases bone mineral density. AIDS. 2009; 23(12):1519-1529. [PubMed: 19531929]

12. [Accessed : 7-1-2011] CDC United States Growth Charts. 2000. In: www.cdc.gov/growthcharts

13. Jacobson DL, Patel K, Siberry GK, Van Dyke RB, DiMeglio LA, Geffner ME, et al. Body fat distribution in perinatally HIV-infected and HIV-exposed but uninfected children in the era of highly active antiretroviral therapy: outcomes from the Pediatric HIV/AIDS Cohort Study. Am J Clin Nutr. 2011; 94(6):1485-1495. [PubMed: 22049166]

14. Caldwell MB, Oxtoby MJ, Simonds RJ, Lindegren ML, Rogers MF. 1994 Revised Classification System for Human Immunodeficiency Virus Infection in Children Less Than 13 Years of Age. MMWR CDC Surveill Summ. 1994; 43:1-10.

15. Greulich, WW.; Pyle, SI. Radiographic Atlas of Skeletal Development of the Hand \& Wrist. Stanford, CA: Stanford University Press; 1959.

16. Ellis, KJ.; Shypailo, RJ. [Accessed : 5-31-2011] Body composition comparison data for children. 2000. In: http://www.bcm.edu/bodycomplab/Applications/zscrmainpage.htm

17. Frost HM. Bone "mass" and the "mechanostat": a proposal. Anat Rec. 1987; 219(1):1-9. [PubMed: 3688455]

18. Bolland MJ, Grey AB, Gamble GD, Reid IR. Clinical Review: Low body weight mediates the relationship between HIV infection and low bone mineral density: a meta-analysis. J Clin Endocrinol Metab. 2007; 92(12):4522-4528. [PubMed: 17925333]

19. Hui SL, DiMeglio LA, Longcope C, Peacock M, McClintock R, Perkins AJ, et al. Difference in bone mass between black and white American children: attributable to body build, sex hormone levels, or bone turnover? J Clin Endocrinol Metab. 2003; 88(2):642-649. [PubMed: 12574194]

20. Rauch F, Plotkin H, DiMeglio L, Engelbert RH, Henderson RC, Munns C, et al. Fracture prediction and the definition of osteoporosis in children and adolescents: the ISCD 2007 Pediatric Official Positions. J Clin Densitom. 2008; 11(1):22-28. [PubMed: 18442750]

21. Hansen AB, Gerstoft J, Kronborg G, Larsen CS, Pedersen C, Pedersen G, et al. Incidence of low and high-energy fractures in persons with and without HIV infection: a Danish population-based cohort study. AIDS. 2012; 26(3):285-293. [PubMed: 22095195]

22. Womack JA, Goulet JL, Gibert C, Brandt C, Chang CC, Gulanski B, et al. Increased risk of fragility fractures among HIV infected compared to uninfected male veterans. PLoS One. 2011; 6(2):e17217. [PubMed: 21359191]

23. Prior J, Burdge D, Maan E, Milner R, Hankins C, Klein M, et al. Fragility fractures and bone mineral density in HIV positive women: a case-control population-based study. Osteoporos Int. 2007; 18(10):1345-1353. [PubMed: 17665239]

24. Siberry GK, Li H, Jacobson D. Fracture risk by HIV infection status in perinatally HIV-exposed children. AIDS Res Hum Retroviruses. 2012; 28(3):247-250. [PubMed: 22471877]

25. Tsekes G, Chrysos G, Douskas G, Paraskeva D, Mangafas N, Giannakopoulos D, et al. Body composition changes in protease inhibitor-naive HIV-infected patients treated with two nucleoside reverse transcriptase inhibitors. HIV Med. 2002; 3(2):85-90. [PubMed: 12010354]

26. van Vonderen MG, Lips P, van Agtmael MA, Hassink EA, Brinkman K, Geerlings SE, et al. First line zidovudine/lamivudine/lopinavir/ritonavir leads to greater bone loss compared to nevirapine/ lopinavir/ritonavir. AIDS. 2009; 23(11):1367-1376. [PubMed: 19424051]

27. Jain RG, Lenhard JM. Select HIV protease inhibitors alter bone and fat metabolism ex vivo. J Biol Chem. 2002; 277(22):19247-19250. [PubMed: 11937496] 
28. Silva CC, Goldberg TB, Teixeira AS, Dalmas JC. The impact of different types of physical activity on total and regional bone mineral density in young Brazilian athletes. J Sports Sci. 2011; 29(3): 227-234. [PubMed: 21170799]

29. [Accessed : 12-7-2011] Panel on Antiretroviral Therapy and Medical Management of HIV-infected Children Guidelines for the Use of Antiretroviral Agents in Pediatric HIV Infection; Nov 8. 2011 p. 1-268.In: http://aidsinfo.nih.gov/ContentFiles/PediatricGuidelines.pdf

30. Purdy JB, Gafni RI, Reynolds JC, Zeichner S, Hazra R. Decreased bone mineral density with offlabel use of tenofovir in children and adolescents infected with human immunodeficiency virus. J Pediatr. 2008; 152(4):582-584. [PubMed: 18346519]

31. Vikulina T, Fan X, Yamaguchi M, Roser-Page S, Zayzafoon M, Guidot DM, et al. Alterations in the immuno-skeletal interface drive bone destruction in HIV-1 transgenic rats. Proc Natl Acad Sci U S A. 2010; 107(31):13848-13853. [PubMed: 20643942]

32. Yin MT, McMahon DJ, Ferris DC, Zhang CA, Shu A, Staron R, et al. Low bone mass and high bone turnover in postmenopausal human immunodeficiency virus-infected women. J Clin Endocrinol Metab. 2010; 95(2):620-629. [PubMed: 19965927]

33. Young B, Dao CN, Buchacz K, Baker R, Brooks JT. Increased Rates of Bone Fracture among HIV-Infected Persons in the HIV Outpatient Study (HOPS) Compared with the US General Population, 2000-2006. Clin Infect Dis. 2011; 52(8):1061-1068. [PubMed: 21398272]

34. Zamboni G, Antoniazzi F, Bertoldo F, Lauriola S, Antozzi L, Tato L. Altered bone metabolism in children infected with human immunodeficiency virus. Acta Paediatr. 2003; 92(1):12-16. [PubMed: 12650292] 
Table 1

Characteristics of HIV-Infected (HIV+) and HIV-Exposed but Uninfected (HEU) Children at the Time of DXA

\begin{tabular}{|c|c|c|c|c|}
\hline \multirow[b]{2}{*}{ Characteristic } & & \multicolumn{3}{|c|}{ HIV+ vs. HEU } \\
\hline & & \multirow{2}{*}{$\frac{\mathbf{H I V}+(\mathbf{N}=\mathbf{3 5 0})}{12.6(10.2,14.4)}$} & \multirow{2}{*}{$\begin{array}{c}\text { HEU (N=160) } \\
10.7(8.9,12.6)\end{array}$} & \multirow{2}{*}{$\frac{\text { p value }^{I}}{<.001}$} \\
\hline Age (years) at DXA & $\operatorname{Median}\left(25^{\text {th }}, 75^{\text {th }}\right)$ & & & \\
\hline Bone age (years) & Median (25th, 75th) & $11.7(10.0,13.5)$ & $11(8.9,13.0)$ & 0.023 \\
\hline \multirow[t]{4}{*}{ Chronological age (CA) vs. bone age (BA) } & $\mathrm{CA}>1 \mathrm{SD}$ of $\mathrm{BA}$ & $62(18 \%)$ & $14(9 \%)$ & $<.001$ \\
\hline & CA within $1 \mathrm{SD}$ of $\mathrm{BA}$ & $169(48 \%)$ & $71(44 \%)$ & \\
\hline & $\mathrm{CA}<1 \mathrm{SD}$ of $\mathrm{BA}$ & $55(16 \%)$ & $55(34 \%)$ & \\
\hline & Tanner 5, BA not expected & $64(18 \%)$ & $20(13 \%)$ & \\
\hline \multirow[t]{2}{*}{ Sex } & Female & $189(54 \%)$ & $84(53 \%)$ & 0.775 \\
\hline & Male & $161(46 \%)$ & $76(48 \%)$ & \\
\hline \multirow[t]{4}{*}{ Race/ethnicity } & Hispanic & $91(26 \%)$ & $60(38 \%)$ & 0.012 \\
\hline & Black Non-Hispanic & $231(66 \%)$ & $84(53 \%)$ & \\
\hline & White/other Non-Hispanic & $27(8 \%)$ & $16(10 \%)$ & \\
\hline & Unknown & $1(0 \%)$ & $0(0 \%)$ & \\
\hline Height Z-score & $\operatorname{Median}\left(25^{\text {th }}, 75^{\text {th }}\right)$ & $-0.3(-1.1,0.4)$ & $0.2(-0.5,0.9)$ & $<.001$ \\
\hline Weight Z-score & $\operatorname{Median}\left(25^{\text {th }}, 75^{\text {th }}\right)$ & $0.1(-0.7,0.9)$ & $0.6(-0.4,1.7)$ & $<.001$ \\
\hline BMI Z-score & $\operatorname{Median}\left(25^{\text {th }}, 75^{\text {th }}\right)$ & $0.2(-0.4,1.2)$ & $0.8(-0.4,1.8)$ & 0.002 \\
\hline \multirow[t]{5}{*}{ Tanner stage } & 1 & $86(25 \%)$ & $56(35 \%)$ & 0.005 \\
\hline & 2 & $73(21 \%)$ & $46(29 \%)$ & \\
\hline & 3 & $59(17 \%)$ & $16(10 \%)$ & \\
\hline & 4 & $68(19 \%)$ & $22(14 \%)$ & \\
\hline & 5 & $64(18 \%)$ & $20(13 \%)$ & \\
\hline \multicolumn{5}{|l|}{ Diet } \\
\hline \multicolumn{5}{|l|}{ Vitamin D (IU/day) } \\
\hline \multirow[t]{3}{*}{$<\mathrm{RDA}$} & no & $137(39 \%)$ & $43(27 \%)$ & 0.028 \\
\hline & yes & $183(52 \%)$ & $94(59 \%)$ & \\
\hline & missing & $30(9 \%)$ & $23(14 \%)$ & \\
\hline Average intake & $\operatorname{Median}\left(25^{\text {th }}, 75^{\text {th }}\right)$ & $125(75,200)$ & $133(91,216)$ & 0.227 \\
\hline \multicolumn{5}{|l|}{ Calcium (mg/day) } \\
\hline \multirow[t]{3}{*}{$<\mathrm{RDA}$} & no & $63(18 \%)$ & $30(19 \%)$ & 0.613 \\
\hline & yes & $257(73 \%)$ & $107(67 \%)$ & \\
\hline & missing & $30(9 \%)$ & $23(14 \%)$ & \\
\hline Average intake & $\operatorname{Median}\left(25^{\text {th }}, 75^{\text {th }}\right)$ & $674(447,990)$ & $681(489,1,004)$ & 0.489 \\
\hline \multirow[t]{3}{*}{ Vitamin supplements } & no & $231(66 \%)$ & $120(75 \%)$ & $<.001$ \\
\hline & yes & $89(25 \%)$ & $17(11 \%)$ & \\
\hline & missing & $30(9 \%)$ & $23(14 \%)$ & \\
\hline \multicolumn{5}{|l|}{ Physical activity } \\
\hline Vigorous physical activity $(\mathrm{min})>75^{\text {th }}$ vs. & no & $187(53 \%)$ & $102(64 \%)$ & 0.624 \\
\hline
\end{tabular}




\begin{tabular}{|lllcl|}
\hline & & \multicolumn{2}{c|}{ HIV+ vs. HEU } \\
\cline { 3 - 4 } Characteristic & & HIV+ (N=350) & HEU (N=160) & p value $^{\mathbf{I}}$ \\
\hline & yes & $59(17 \%)$ & $37(23 \%)$ \\
& missing & $104(30 \%)$ & $21(13 \%)$ \\
\hline
\end{tabular}

${ }^{l}$ Fisher's exact test for categorical variables and Wilcoxon for continuous variables

Abbreviations: Children with perinatal HIV infection (HIV+), Children who were perinatally exposed but uninfected (HEU), Dual energy X-ray absorptiometry (DXA), Standard deviation (SD), Chronological age (CA), Bone age (BA), percentile (\%ile), Recommended dietary allowance (RDA) 
Table 2

Disease Severity Characteristics of HIV+ Children at the Time of DXA

\begin{tabular}{|llc|}
\hline Characteristic & & HIV+ (N=350) \\
\hline CDC clinical category & N/A & $166(47 \%)$ \\
& B & $98(28 \%)$ \\
& C & $86(25 \%)$ \\
CD4 count (cells/mm $)$ & Median (25th, 75th) & $725(521,934)$ \\
CD4 category (cells/mm $\left./ \mathrm{mm}^{3}\right)$ & $<200$ & $10(3 \%)$ \\
& $200-500$ & $69(20 \%)$ \\
& $>500$ & $270(77 \%)$ \\
& Missing & $1(0 \%)$ \\
CD4\% & Median (25th, 75th) & $33(27,38)$ \\
CD4\% category & $0-14 \%$ & $15(4 \%)$ \\
& $15 \%-24 \%$ & $57(16 \%)$ \\
& $>=25 \%$ & $278(79 \%)$ \\
Nadir CD4\% & Median (25th, 75th) & $20(14,26)$ \\
HIV viral load (copies/mL) & $<400$ & $194(55 \%)$ \\
& $401-5000$ & $102(29 \%)$ \\
& $>5000$ & $54(15 \%)$ \\
\hline
\end{tabular}

Abbreviations: Dual energy X-ray absorptiometry (DXA), Centers for Disease Control (CDC) 


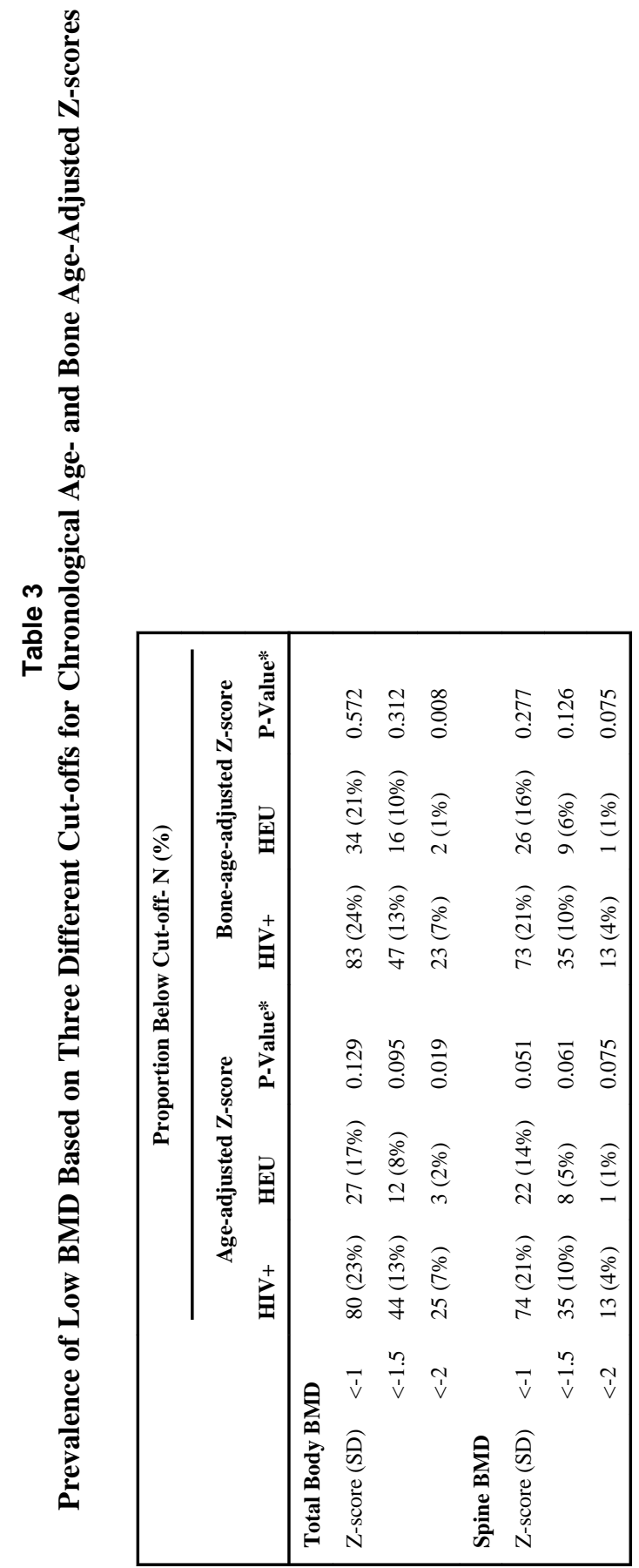

AIDS. Author manuscript; available in PMC 2014 September 08. 


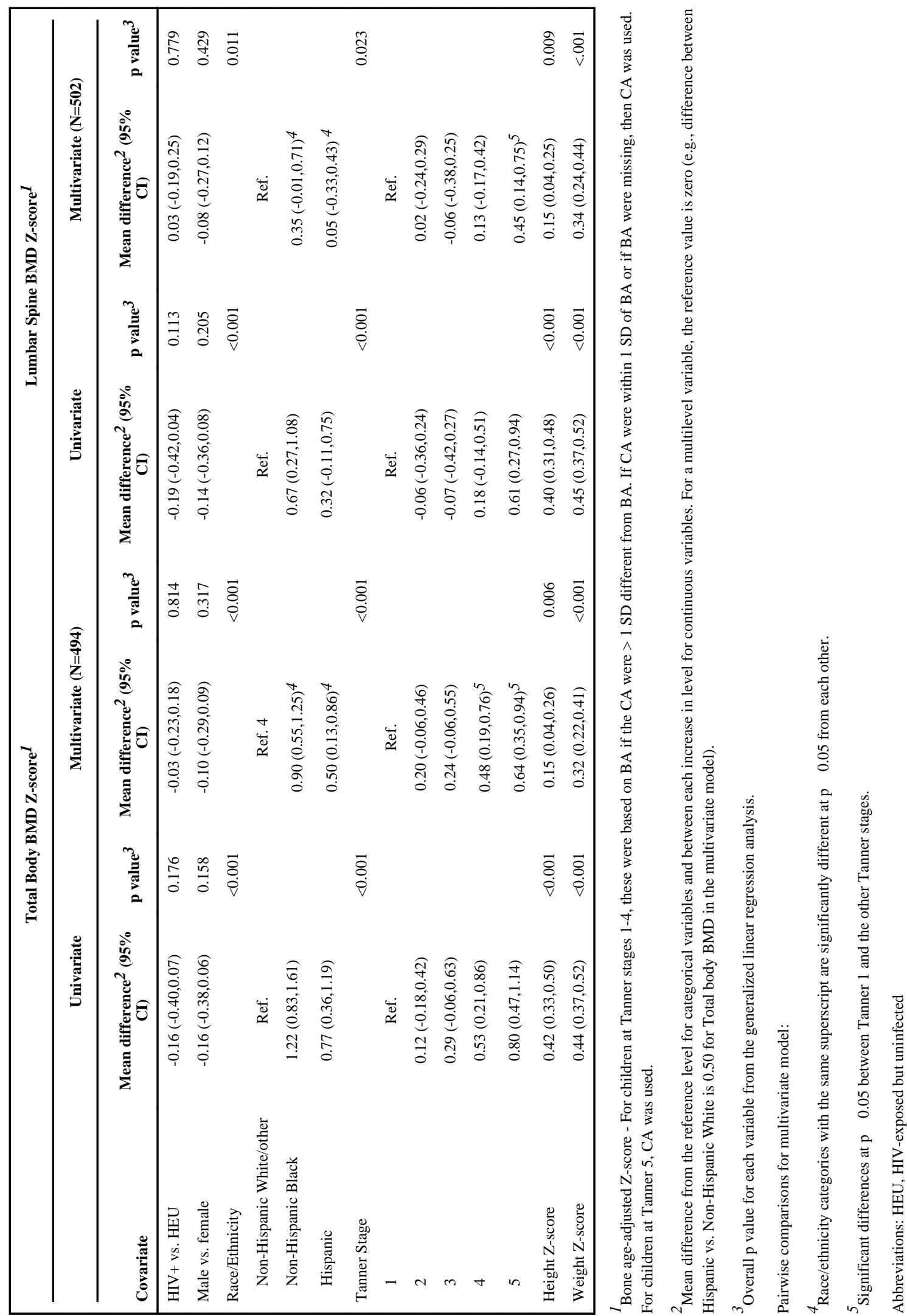


Table 5

Multivariate Models of Total Body and Spine BMD Z-scores in HIV-infected Children

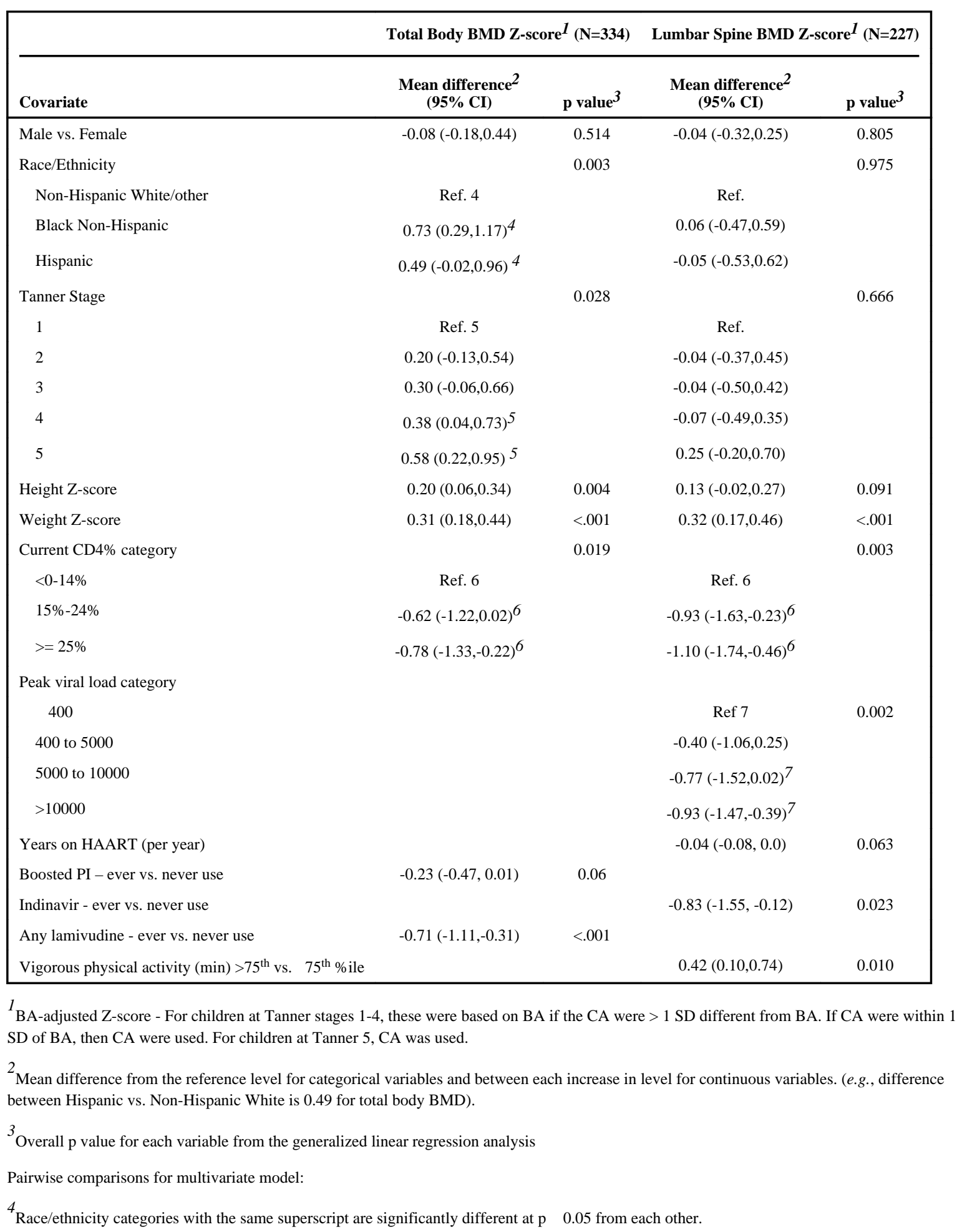


${ }^{5-7}$ Significant differences at $\mathrm{p} \leq 0.05$ between reference levels and other levels. Tanner levels 2-5 were compared separately with Tanner 1. Current CD4\% category levels were compared with reference level $(<15 \%)$. Peak viral load levels were compared with reference level $(\leq 400)$. 